\title{
Emissão de metano por decomposição de resíduo florestal inundado
}

\author{
Maria M. V. Zanoni ${ }^{1}$, Josiléia A. Zanatta ${ }^{2}$, Jeferson Dieckow ${ }^{3}$, Akemi $\operatorname{Kan}^{4}$ \& Carlos B. Reissmann ${ }^{5}$
}

Palavras-chave:
solo alagado
fitomassa
decomposição anaeróbica
metanogênese
gases do efeito estufa

Key words:

flooded soil

biomass

anaerobic decomposition

methanogenesis

greenhouse gases

\begin{abstract}
R E S U M O
A construção de represas pode aumentar a emissão de gases do efeito estufa (GEE), principalmente metano $\left(\mathrm{CH}_{4}\right)$ pela decomposição anaeróbica dos resíduos florestais, como galhos, ramos, folhas e miscelânea inundados. Objetivou-se, neste estudo, avaliar a emissão de $\mathrm{CH}_{4}$ após a inundação do solo coberto com resíduos florestais. Unidades experimentais foram construídas com tubos de PVC contendo solo e diferentes combinações de dose ( 0 ; 21,2; 42,3 e 64,1 $\mathrm{Mg} \mathrm{ha}^{-1}$ ) e tipo de resíduos (folhas, ramos e miscelânea, galhos e composição original) e água de rio. $\mathrm{O}$ delineamento experimental foi inteiramente casualizado com arranjo fatorial e três repetições. As taxas de emissão de $\mathrm{CH}_{4}$ foram monitoradas em 19 eventos durante um ano (Fevereiro/2012 a Março/2013). Cerca de 75 dias após a incubação do solo com resíduos florestais verificou-se aumento das emissões de $\mathrm{CH}_{4}$, ocorrendo dois picos de emissão, aos 111 e aos 249 dias. A emissão acumulada de $\mathrm{CH}_{4}$ no primeiro ano de alagamento foi de $200 \mathrm{~g} \mathrm{C} \mathrm{m}^{-2}$ na dose zero, passando a valores próximos a $400 \mathrm{~g} \mathrm{C} \mathrm{m}^{-2}$ nas doses de 21,2 $\mathrm{Mg} \mathrm{ha}^{-1}$ e maiores, não havendo efeito do tipo de resíduo, apenas da dose, como fator isolado.
\end{abstract}

\section{Methane emission by decomposition of flooded forest residues}

\begin{abstract}
A B S T R A C T
The construction of dams can increase the emission of greenhouse gases (GHG), mainly methane $\left(\mathrm{CH}_{4}\right)$ by the anaerobic decomposition of forest residues like twigs, branches, leaves and miscellaneous flooded. The aim of this study was to evaluate the emission of $\mathrm{CH}_{4}$ after the flooding of soil covered with residuals of forests. Experimental units were built with PVC tubes containing soil covered with different combinations of dose $(0 ; 21.2$; 42.3 and $64.1 \mathrm{Mg} \mathrm{ha}^{-1}$ ) and type (branches, leaves and miscellaneous; twigs; and original composition) of forest residues and river water. The experimental design was completely randomized with factorial arrangement and three replications. Rates of $\mathrm{CH}_{4}$ emission were monitored in 19 events during one year (February/2012 to March/2013). Approximately, 75 days after incubation of soil with residue, a significant increase in $\mathrm{CH}_{4}$ emission was observed occurring two emission peaks: at 111 and 249 days. The cumulative emission of $\mathrm{CH}_{4}$ in the first year after flooding was $200 \mathrm{~g} \mathrm{C} \mathrm{m}^{-2}$ in the dose zero, until above $400 \mathrm{~g} \mathrm{C}$ $\mathrm{m}^{-2}$ in the $21.2 \mathrm{Mg} \mathrm{ha}^{-1}$ and higher doses, with no effect of type of residues, only the dose as an isolated factor.
\end{abstract}

Protocolo 020.14 - 17/01/2014 • Aprovado em 19/09/2014 • Publicado em 01/02/2015

${ }^{1}$ PPGCS/UFPR. Curitiba, PR. E-mail: mariamartavz@gmail.com (Autora correspondente)

${ }^{2}$ CNPF/EMBRAPA. Colombo, PR. E-mail: josileia.zanatta@embrapa.br

${ }^{3}$ PPGCS/UFPR. Curitiba, PR. E-mail: jefersondieckow@ufpr.br

${ }^{4}$ CEHPAR/LACTEC. Curitiba, PR. E-mail: akemi.kan@hotmail.com

${ }^{5}$ PPGCS/UFPR. Curitiba, PR. E-mail: karlbreis@yahoo.com.br 


\section{INTRODUÇÃo}

Nas últimas duas décadas tem-se pesquisado, de forma mais intensa, sobre a emissão dos gases do efeito estufa (GEE) pelos reservatórios de usinas hidrelétricas, com maior enfoque nas emissões de metano $\left(\mathrm{CH}_{4}\right)$ (Rudd et al., 1993; Santos et al., 2008). $\mathrm{O} \mathrm{CH}_{4}$ é produzido no fundo dos reservatórios pela decomposição anaeróbica da matéria orgânica e emitido na interface água-ar, através de bolhas ou difusão. Apesar dos inúmeros estudos ainda há incertezas em relação à quantidade, à variação temporal, às fontes e contribuição líquida dos reservatórios de hidrelétricas à emissão de $\mathrm{CH}_{4}$ (Louis et al., 2000; Fearnside, 2008). Tal dúvida persiste principalmente pela escassez de estudos sistemáticos e abrangentes nesses ambientes (Santos et al., 2008).

No Brasil, o segundo inventário nacional de emissão de GEE identificou que, em 2005, as principais fontes de GEE foram a agropecuária (emissão de $87 \%$ do $\mathrm{N}_{2} \mathrm{O}$ emitido no país e $90 \%$ do $\left.\mathrm{CH}_{4}\right)$, as mudanças no uso da terra $\left(76 \%\right.$ do $\left.\mathrm{CO}_{2}\right)$ e a geração de energia $\left(19,2 \%\right.$ do $\mathrm{CO}_{2}, 3 \%$ do $\mathrm{CH}_{4}, 2,2 \%$ do $\mathrm{N}_{2} \mathrm{O}$ ), sobremaneira pela queima imperfeita de combustíveis e também devido à fuga de $\mathrm{CH}_{4}$ durante os processos de produção e transporte de gás natural e mineração de carvão. $\mathrm{O}$ inventário apontava que, na época, esta última fonte respondia pela emissão de $328,8 \mathrm{Gg} \mathrm{CO}_{2}$ equivalente, ou seja, $15 \%$ do potencial de aquecimento global do país. Além de que de 1990 a 2005 foi registrado, para esta fonte, um aumento de 74,3\% da emissão de $\mathrm{CO}_{2}, 26,7 \%$ de $\mathrm{CH}_{4}$ e $42,9 \%$ de $\mathrm{N}_{2} \mathrm{O}$, o que reforça o fato de que, apesar da matriz energética do país ser considerada "limpa" pela participação das fontes renováveis, pesquisas têm revelado que o setor contribui significativamente para as emissões de GEE (MCT, 2010).

Na emissão de GEE por reservatórios de usinas hidrelétricas o carbono dos gases emitidos tem origem na decomposição de três fontes principais - biomassa original inundada, biomassa formada pela fotossíntese e biomassa alóctone. Buscou-se então, neste estudo, abordar a emissão por apenas uma dessas fontes, a biomassa original inundada, a fim de se avaliar relações entre esses dois parâmetros (emissão vs. biomassa inundada), considerando que tanto a quantidade dessa biomassa como sua composição terão influência sobre a emissão visto que a decomposição da matéria orgânica também depende desses fatores (Santos et al., 2008; Sbrissia et al., 2011).

A decomposição da biomassa inundada é considerada a principal fonte de emissão de gases em reservatórios nos primeiros anos após o alagamento (Abril et al., 2005). A magnitude deste processo pode ser influenciada por variáveis ambientais e pelas variáveis de qualidade da matéria orgânica morta da floresta inundada como teor de $\mathrm{N}$, relação $\mathrm{C} / \mathrm{N}$, lignina:N, concentração de fósforo, (Lee \& Bukaveckas, 2002; Neiff et al., 2006), entre outras.

Propôs-se neste trabalho avaliar a emissão de $\mathrm{CH}_{4}$ e a relação com atributos químicos e físicos da água, desde que refletissem a qualidade/quantidade do resíduo inundado, simulando o alagamento de resíduos vegetais na construção de reservatórios de hidrelétricas.

\section{Material e Métodos}

Resíduos vegetais e solo foram coletados em aproximadamente 2 ha de uma área recém-desmatada de floresta secundária a ser alagada pelo reservatório de uma usina hidrelétrica construída no Rio Tibagi, município de Telêmaco Borba, PR. A área, situada entre as latitudes $24^{\circ} 10^{\prime} 34^{\prime \prime} \mathrm{S}$ e $24^{\circ} 10^{\prime} 37^{\prime \prime} \mathrm{S}$ e longitudes $50^{\circ} 39^{\prime} 46^{\prime \prime} \mathrm{O}$ e $50^{\circ} 39^{\prime} 44^{\prime \prime} \mathrm{O}$ tinha, como vegetação nativa, a transição de Floresta Ombrófila Mista e Floresta Estacional Semidecidual. Troncos e galhos > $8 \mathrm{~cm}$ de diâmetro já haviam sido removidos pela operação de supressão da floresta. Doze parcelas de amostragem de 2 × $2 \mathrm{~m}$ foram delimitadas e coletados todos os seus resíduos vegetais, sendo classificados em folhas e miscelânea (material vegetal particulado), ramos (de diâmetro menor que $2 \mathrm{~cm}$ ) e galhos (de diâmetro entre 2 e $8 \mathrm{~cm}$ ). Em cada parcela foram coletadas amostras de solo com o auxílio de um gabarito de 25 x $50 \mathrm{~cm}$ nas profundidades de 0 a $5 \mathrm{~cm}$ e de 5 a $10 \mathrm{~cm}$. As amostras vegetais e de solo foram pesadas a campo e a partir de então reservadas para constituir as amostras compostas utilizadas para a montagem das unidades experimentais. Uma porção das amostras de solo e dos resíduos já classificados foi secada em estufa para posteriores análises químicas e físicas.

As amostras compostas dos resíduos vegetais foram analisadas em duplicata para os teores de lignina, celulose, hemicelulose e extrativos (Soest \& Wine, 1968) e em triplicata as concentrações de N, C, P, K, Ca e Mg (Martins \& Reissmann, 2007). A densidade e a umidade gravimétrica do solo foram calculadas para cada uma das 12 parcelas e camadas de coleta. A granulometria foi obtida pelo método da pipeta. Dentre as análises químicas do solo foram realizadas as análises de $\mathrm{pH}$ (em água, $\mathrm{CaCl}_{2}$ e SMP); carbono orgânico, $\mathrm{Ca}^{2+}, \mathrm{Mg}^{2+}$ e $\mathrm{Al}^{3+}$ e $\mathrm{K}^{+}$trocáveis; CTC e P disponível. Todas as avaliações acima foram realizadas conforme métodos descritos em EMBRAPA (1997); já o N-total do solo foi obtido pelo método Kjeldahl e o C-total pelo método Nelson \& Sommers (1996).

As unidades consistiram de tubos de PVC com $150 \mathrm{~mm}$ de diâmetro e 2,95 m de altura, dispostas na vertical. Na porção mais inferior do tubo foram rearranjadas as camadas de 0-5 e $5-10 \mathrm{~cm}$ de solo, seguidas pela camada de resíduos florestal cuja altura variou conforme a dose de resíduos florestais testada; posteriormente, o tubo foi preenchido com água de rio até 2,8 m de altura.

As unidades experimentais se constituíram da combinação de três doses de resíduos florestais $\left(21,2,42,3\right.$ e $64,1 \mathrm{Mg} \mathrm{ha}^{-1}$, respectivamente, 33,66 e $100 \%$ da dose coletada em campo) e três tipos de resíduos (apenas folhas e ramos menores que $2 \mathrm{~cm}$ de diâmetro - FR, composição original - ORIG, galhos entre $2 \mathrm{e}$ $8 \mathrm{~cm}$ de diâmetro - GG), aplicados sobre o solo e submersos em água, havendo um tratamento testemunha constituído apenas de solo e água. As nove combinações, além do tratamento testemunha, foram monitoradas em três repetições.

A água para enchimento dos tubos foi coletada no rio Iraí, na estação 65006075 da SUDERHSA, no município de Pinhais$\mathrm{PR}$, e não no rio Tibagi, devido a problemas logísticos. Uma amostra da água foi caracterizada inicialmente, em 16/02/2012, conforme metodologia de APHA (1998) para as seguintes variáveis: $\mathrm{pH}$ (atráves de $\mathrm{pH}$-metro digital WTM - modelo

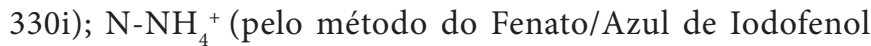
- Colorimetria); N-NO ${ }_{2}^{-}$(método da Sulfanilamida /N-1-

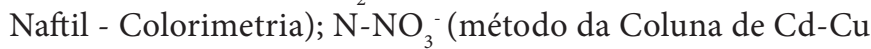
-Colorimetria) e COD (Combustão a alta temperatura em analisador TOC-VCPH Shimadzu). Ao longo do experimento 
a qualidade da água dos tubos foi avaliada aos 10, 33, $137 \mathrm{e}$ 242 dias após preenchimento dos tubos para monitorar o comportamento do nitrato, amônio, nitrito. As amostras de água foram retiradas da porção inferior do tubo através de uma torneira instalada a $30 \mathrm{~cm}$ de altura.

A amostragem de emissões gasosas nas unidades experimentais foi realizada de fevereiro de 2012 a março de 2013 em 19 coletas, aos: 0, 7, 12, 18, 34, 45, 55, 62, 76, 90, 111, $136,172,188,227,249,292,320$ e 377 dias após o alagamento dos resíduos florestais e solo. Na coleta e na quantificação do $\mathrm{CH}_{4}$ foi adotada uma adaptação ao método das câmaras estáticas propostas por Parkin et al. (2003). Como câmaras foram utilizadas tampas de PVC (CAPs) encaixadas no momento da coleta na porção superior do tubo, nas quais havia um orifício central justamente fechado com prensacabo e mangueira de plástico justaposta, em que foi adaptada válvula de três posições, que acopla a seringa no momento da coleta. Após o fechamento da câmara as amostras de ar foram coletadas com seringas de polipropileno de $20 \mathrm{~mL}$, em três tempos de amostragem. A concentração do $\mathrm{CH}_{4}$ na amostra de ar foi obtida por cromatografia gasosa, em equipamento Trace GC Ultra, com forno a $70{ }^{\circ} \mathrm{C}$ e detector FID.

A taxa de emissão de $\mathrm{CH}_{4}$ foi obtida pela inclinação da reta que corresponde à regressão linear dos valores de concentração de $\mathrm{CH}_{4}$ obtida nas amostras de ar versus os tempos de medição $(0,15$ e $30 \mathrm{~min})$. A emissão acumulada de $\mathrm{CH}_{4}$ foi obtida pela integração das taxas de emissão diárias ao longo dos 377 dias (Parkin et al., 2003).

O delineamento experimental foi inteiramente casualizado com fatorial e três repetições. $\mathrm{Na}$ análise ao longo do tempo foram considerados três fatores: 3 tipos de resíduo $\mathrm{x} 4$ doses $\mathrm{x}$ 19 coletas e na parte referente à emissão acumulada de $\mathrm{CH}_{4}$, dois fatores: 3 tipos $\mathrm{x} 4$ doses. A testemunha foi considerada a dose zero de resíduos.

A análise de variância foi empregada a 5\% de significância. Para a emissão do $\mathrm{CH}_{4}$ ao longo do tempo e as variáveis de qualidade da água foi realizada a comparação entre médias pelo teste de Tukey e o teste da correlação de Pearson. Já para a emissão anual acumulada de $\mathrm{CH}_{4}$ foi proposto ajuste a um modelo de regressão. Este ajuste foi realizado considerandose os três tipos de resíduo para cada dose e as três repetições, totalizando nove valores referentes a cada dose.

\section{Resultados e Discussão}

O solo foi caracterizado nas camadas de 0 a $5 \mathrm{~cm}$ e 5 a $10 \mathrm{~cm}$ (Tabela 1). Respectivamente, tais camadas possuíam densidade de 1140 e $1400 \mathrm{~kg} \mathrm{~m}^{-3}$, umidade gravimétrica de 24,8 e 21,2\%, 260 e $240 \mathrm{~g} \mathrm{~kg}^{-1}$ de argila. As amostras foram mantidas refrigeradas entre a coleta e a montagem das camadas de solo nos tubos de PVC, adotando-se a densidade e a umidade do solo original. Segundo a CQFS (2004) os indicadores de acidez ( $\mathrm{pH}$ água, $\mathrm{Al}^{+3}, \mathrm{H}+\mathrm{Al}^{+3}, \mathrm{SB}, \mathrm{m}$ ) configuram valores muito baixos, $\mathrm{SB}$, valor $\mathrm{T}$ e matéria orgânica medianos, $\mathrm{Ca}^{+2}, \mathrm{Mg}^{+2}$ e $\mathrm{P}$ baixo e $\mathrm{K}$ alto.

A quantidade de resíduos florestais na superfície estimada na média de 12 parcelas, foi de $64,1 \mathrm{Mg} \mathrm{ha}^{-1}$, formada por: $24,9 \%$ folhas e miscelânea, 20,0\% ramos e 55,1\% galhos. A qualidade química da composição original e das demais combinações dos tipos de resíduos consta na Tabela 2.
Tabela 1. Características químicas das duas camadas superficiais do solo e da água

\begin{tabular}{lcc}
\hline \multirow{2}{*}{ Atributos } & \multicolumn{2}{c}{ Camadas (cm) } \\
\cline { 2 - 3 } Densidade aparente, $\mathrm{kg} \mathrm{m}^{-3}$ & $\mathbf{0 - 5}$ & $\mathbf{5 - 1 0}$ \\
Umidade gravimétrica, $\mathrm{kg} \mathrm{kg}^{-1}$ & $0,248 \pm 0,0411$ & $0,212 \pm 0,0243$ \\
\hline Teor de argila, $\mathrm{g} \mathrm{kg}^{-1}$ & 260 & 240 \\
Teor de silte, $\mathrm{g} \mathrm{kg}^{-1}$ & 120,5 & 132,5 \\
$\mathrm{pH}$ água & $4,54 \pm 0,03$ & $4,13 \pm 0,06$ \\
$\mathrm{Al}^{+3}$ trocável, $\mathrm{cmol}_{\mathrm{c}} \mathrm{dm}^{-3}$ & $1,57 \pm 0,03$ & $2,38 \pm 0,02$ \\
$\mathrm{H}+\mathrm{Al}^{+3}, \mathrm{cmol}_{\mathrm{c}} \mathrm{dm}^{-3}$ & $9,33 \pm 0,28$ & $9,73 \pm 0,04$ \\
$\mathrm{Ca}^{+2}, \mathrm{cmol}_{\mathrm{c}} \mathrm{dm}^{-3}$ & $1,62 \pm 0,04$ & $0,54 \pm 0,01$ \\
$\mathrm{Mg}^{+2}, \mathrm{cmol}_{\mathrm{c}} \mathrm{dm}^{-3}$ & $0,48 \pm 0,03$ & $0,18 \pm 0,09$ \\
$\mathrm{~K}^{+} \mathrm{cmol}_{\mathrm{c}} \mathrm{dm}^{-3}$ & $0,21 \pm 0,00$ & $0,11 \pm 0,00$ \\
$\mathrm{SB}, \mathrm{cmol}_{\mathrm{cm}} \mathrm{dm}^{-3}$ & $2,3 \pm 0,01$ & $0,83 \pm 0,09$ \\
$\mathrm{Valor} \mathrm{T}_{\mathrm{cmol}} \mathrm{dm}^{-3}$ & $11,63 \pm 0,27$ & $10,55 \pm 0,09$ \\
$\mathrm{P}, \mathrm{mg} \mathrm{kg}^{-1}$ & $7,02 \pm 0,75$ & $3,59 \pm 0,36$ \\
$\mathrm{C}, \mathrm{g} \mathrm{kg}^{-1}$ & $24,51 \pm 1,64$ & $17,29 \pm 0,56$ \\
$\mathrm{~N}, \mathrm{~g} \mathrm{~kg}^{-1}$ & $2,20 \pm 0,35$ & $2,68 \pm 0,18$ \\
$\mathrm{~V}, \%$ & $19,82 \pm 0,56$ & $7,85 \pm 0,82$ \\
$\mathrm{~m}, \%$ & $40,54 \pm 0,46$ & $74,22 \pm 2,05$ \\
\hline
\end{tabular}

Tabela 2. Caracterização química dos três tipos de resíduos florestais

\begin{tabular}{|c|c|c|c|}
\hline \multirow{2}{*}{ Parâmetros } & $\mathrm{FR}^{*}$ & ORIG* & $\mathbf{G G}^{*}$ \\
\hline & \multicolumn{3}{|c|}{$\left(g^{~ k g}{ }^{-1}\right)$} \\
\hline Lignina & $25,78 \pm 0,45$ & $23,06 \pm 0,55$ & $20,85 \pm 0,64$ \\
\hline Celulose & $55,01 \pm 0,29$ & $56,90 \pm 0,16$ & $58,45 \pm 0,06$ \\
\hline Hemicelulose & $14,00 \pm 0,54$ & $13,95 \pm 0,25$ & $13,92 \pm 0,01$ \\
\hline Extrativos & $3,62 \pm 0,20$ & $3,45 \pm 0,33$ & $3,31 \pm 0,44$ \\
\hline $\mathrm{P}$ & $0,88 \pm 0,08$ & $0,63 \pm 0,06$ & $0,42 \pm 0,05$ \\
\hline K & $2,19 \pm 0,11$ & $2,03 \pm 0,15$ & $1,90 \pm 0,17$ \\
\hline $\mathrm{Ca}$ & $2,36 \pm 0,45$ & $2,88 \pm 0,36$ & $3,30 \pm 0,28$ \\
\hline $\mathrm{Mg}$ & $1,06 \pm 0,04$ & $0,80 \pm 0,03$ & $0,58 \pm 0,03$ \\
\hline C & $442,51 \pm 1,48$ & $440,68 \pm 0,97$ & $439,20 \pm 0,56$ \\
\hline N & $8,95 \pm 0,30$ & $6,46 \pm 0,17$ & $4,43 \pm 0,06$ \\
\hline $\mathrm{C} / \mathrm{N}$ & $49,47 \pm 1,84$ & $68,25 \pm 1,92$ & $99,07 \pm 1,42$ \\
\hline Lignina/N & $2,88 \pm 0,15$ & $3,57 \pm 0,18$ & $4,70 \pm 0,20$ \\
\hline
\end{tabular}

*FR - Folhas e ramos (ramos $<2,0 \mathrm{~cm}$ diâmetro, folhas e miscelânea); ORIG - Composição original (todos os tipos de resíduos conforme a proporção obtida na amostragem em campo) e GG - Galhos (ramos com diâmetro entre 2 e $8 \mathrm{~cm}$ )

A amostra inicial de água possuía $\mathrm{pH}=7,3 ; \mathrm{N}_{-} \mathrm{NH}_{4}^{+}=1,75$ $\mathrm{mg} \mathrm{L}^{-1} ; \mathrm{N}^{-\mathrm{NO}_{2}}{ }^{-}=0,28 \mathrm{mg} \mathrm{L}^{-1} ; \mathrm{N}^{-\mathrm{NO}_{3}}=0,37 \mathrm{mg} \mathrm{L}^{-1}$ e COD $=$ $4,283 \mathrm{mg} \mathrm{L}^{-1}$.

Para a emissão de $\mathrm{CH}_{4}$ não houve interação das campanhas de coleta com o tipo de resíduo nem com a dose avaliada. O comportamento das emissões médias por tipo de resíduo e dose, ao longo do tempo, consta na Figura $1 \mathrm{~A}$ e $1 \mathrm{~B}$, respectivamente. A média referente a todos os tipos e doses de resíduos por campanha e a diferença mínima significativa (dms) entre cada campanha estão contidas na Figura 1C.

Durante o experimento as taxas médias de emissão de $\mathrm{CH}_{4}$ variaram de $-0,00260$ a $113 \mathrm{mg} \mathrm{C} \mathrm{m}^{-2} \mathrm{~h}^{-1}$, porém a maioria se concentrou entre 3,51 e $43,3 \mathrm{mg} \mathrm{C} \mathrm{m}^{-2} \mathrm{~h}^{-1}$, sendo a mediana igual a 25,9 $\mathrm{mg} \mathrm{C} \mathrm{m}^{-2} \mathrm{~h}^{-1}$. A emissão de $\mathrm{CH}_{4}$ é o resultado líquido entre o processo de produção de $\mathrm{CH}_{4}$, a metanogênese, e de absorção, a metanotrofia. A ocorrência de taxas negativas de emissão de $\mathrm{CH}_{4}$ nas primeiras medições (0-7 dias) indica que maior quantidade de $\mathrm{CH}_{4}$ foi consumida pelo ambiente do que aquele que foi efetivamente produzido. Este comportamento esteve, provalvemente, relacionado à atividade de bactérias metanotróficas e à fase de adaptação de grupos metanogênicos. O solo coletado para o desenvolvimento do estudo se encontrava numa condição aerada, possivelmente com alta 


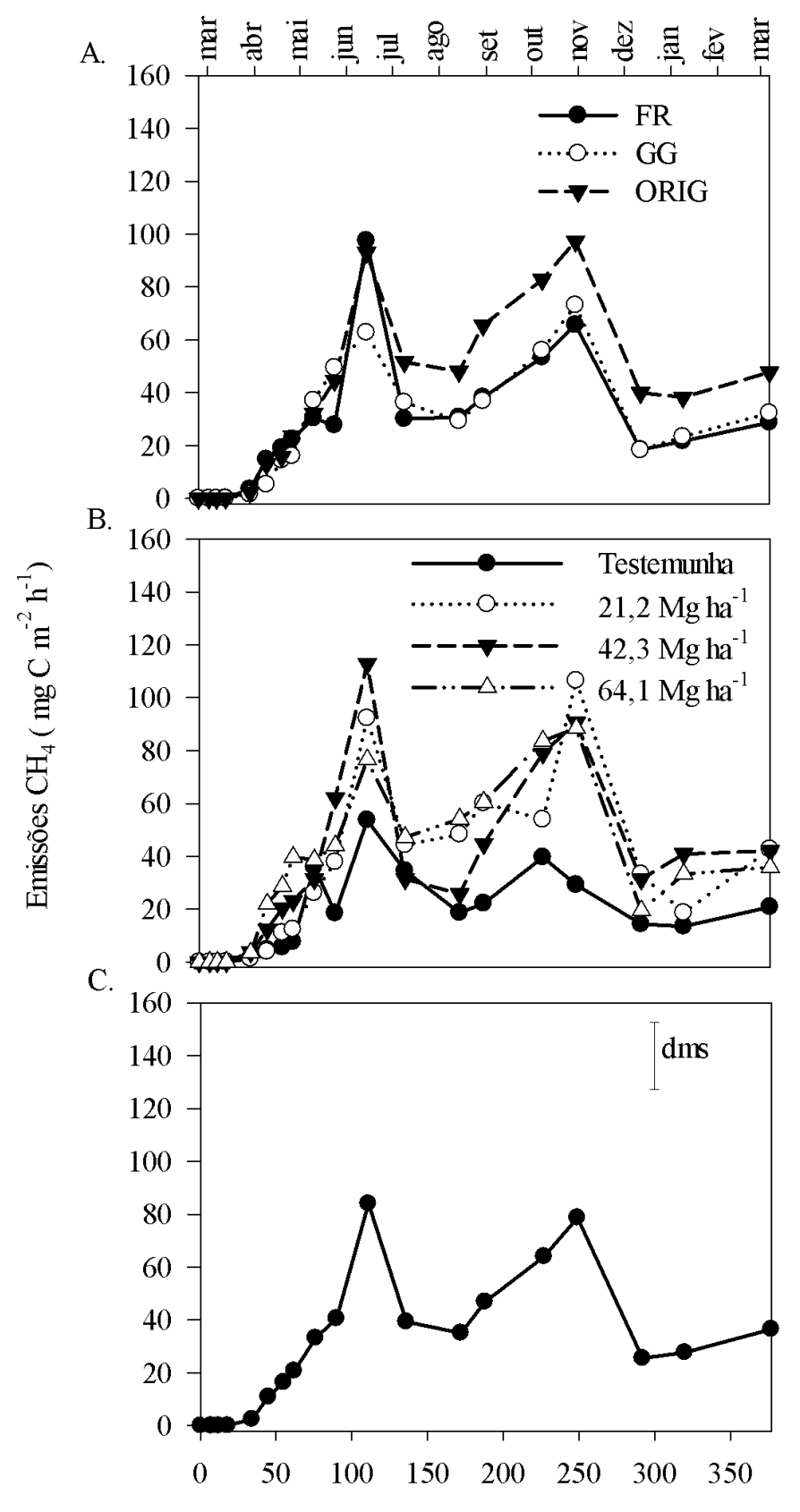

Dias após o alagamento (d)

Figura 1. Emissão de $\mathrm{CH}_{4}$ ao longo do tempo por tipo de resíduo florestal $(\mathrm{A})$, por dose $(\mathrm{B})$ e a média geral, com a barra representando a diferença mínima significativa (dms) entre campanhas $(\mathrm{C})$

atividade metanotrófica e baixa metanogênese; contudo, no decorrer do estudo as condições foram desfavoráveis ao metabolismo metanotrófico e favoráveis à metanogênese predominando, a partir daí, a emissão líquida de $\mathrm{CH}_{4}$.

Em relação à faixa de valores na qual se concentrou a maioria das taxas deste experimento, pode ser considerada elevada se comparada às medidas realizadas em 11 reservatórios no país cujos valores observados não ultrapassaram, na maioria das vezes, 3,0 $\mathrm{mg} \mathrm{C} \mathrm{m}^{-2} \mathrm{~h}^{-1}$ (Santos et al., 2008), sendo o maior valor verificado num lago que havia sido preenchido há 2 anos. A maior emissão observada neste estudo é condizente com áreas inundadas recentemente em que toda a biomassa alagada é fonte de $\mathrm{CH}_{4}$. Com o passar dos anos a contribuição desta fonte às emissões tende a diminuir (Fearnside, 2008). Além do mencionado acima, outro fator que pode ter contribuído para maximizar as taxas de emissão de $\mathrm{CH}_{4}$ neste estudo é a pequena altura da coluna de água permitindo que praticamente todo o $\mathrm{CH}_{4}$ produzido atinja a superfície. Fearnside (2008) calculou, utilizando dados de Galy-Lacaux et al. (1999) a emissão relativa que seria observada em diferentes profundidades do reservatório tendo como base a emissão a $30 \mathrm{~m}$ profundidade. $\mathrm{O}$ autor verificou que a profundidade de $0-3 \mathrm{~m}$ emitiu $87 \%$ mais $\mathrm{CH}_{4}$ do que a camada de 7-8 $\mathrm{m}$ de profundidade, o que é atribuído à menor temperatura da camada de 7-8 $\mathrm{m}$ afetando a pressão e a solubilidade do $\mathrm{CH}_{4}$.

$\mathrm{Na}$ análise temporal da emissão de $\mathrm{CH}_{4}$ se destacam dois comportamentos: (i) período inicial com baixa emissão média e (ii) a ocorrência de picos. Este comportamento é descrito por Bianchini Jr. et al. (2010) quando do estudo de emissões de $\mathrm{CH}_{4}$ e $\mathrm{CO}_{2}$ a partir da decomposição de macrófitas de uma lagoa tropical em condições controladas de anaerobiose, temperatura e sem luminosidade. Os autores definiram três estágios na metanogênese: (i) Fase Lag ( $\mathrm{CH}_{4}$ não era produzido); (ii) $\mathrm{CH}_{4}$ começa a ser produzido, alcançando taxas máximas e (iii) decréscimo nas taxas até chegar a zero. Neste trabalho as duas primeiras fases deste processo foram observadas; entretanto, não é possível afirmar que a fase 3 foi atingida. A fase Lag ou o período de baixa emissões coincide com a redução das formas oxidadas de $\mathrm{N}$ na água (Figura $2 \mathrm{~A}$ e B), apresentando correlação fraca e negativa com as emissões de $\mathrm{CH}_{4}(\mathrm{~N}$ $\left.\mathrm{NO}_{2}{ }^{-} \mathrm{r}=-0,35 ; \mathrm{N}^{-} \mathrm{NO}_{3}{ }_{3}^{-} \mathrm{r}=-0,27\right)$. Na presença desses íons a metanogênese é inibida pela competição pelo carbono orgânico dissolvido e $\mathrm{H}_{2}$ com denitrificadores e efeitos tóxicos de óxidos de nitrogênio (Andalib et al., 2011). $\mathrm{NO}_{2}^{-}$em concentrações de $1,4 \mathrm{mg} \mathrm{L}^{-1}$ foi capaz de inibir em $50 \%$ a atividade de grupos metanogênicos (Klüber \& Conrad, 1998).

Após 75 dias de alagamento as emissões médias passam a ser significativamente maiores que zero. Nesta fase ocorre a degradação inicial do substrato que inclui a adaptação das bactérias ao meio e antecede a emissão de $\mathrm{CH}_{4}$ (Bianchini et al., 2010). Embora nesse tempo tenha ocorrido a formação de substrato para a formação de $\mathrm{CH}_{4}$, também se deve considerar que a própria água já continha certa quantidade de matéria orgânica dissolvida, a qual estava mais facilmente disponível às bactérias e deve ter sido a principal fonte das primeiras emissões de $\mathrm{CH}_{4}$ à atmosfera (Antonio \& Bianchini Jr., 2003; Gimenes et al., 2010).

Quanto à ocorrência dos picos de emissão alcançando valores próximos a $80 \mathrm{mg} \mathrm{C} \mathrm{m}^{-2} \mathrm{~h}^{-1}$ (Figura $3 \mathrm{C}$ ), deve estar relacionada a ciclos de crescimento das bactérias. O primeiro pico, aos 111 dias, ocorre no início do inverno com temperatura do ar em elevação (entre 10 e $15^{\circ} \mathrm{C}$ ), mas decaindo rapidamente na sequência. Este evento gerou, possivelmente, efeito negativo na população bacteriana (Rittmann \& McCarty, 2001) e mais especificamente nas bactérias metanogênicas (Das \& Adhya, 2012), além de ter desfavorecido a geração de $\mathrm{CH}_{4}$ (Bianchini Jr. et al., 2010). Segundo Kotzé et al. (1969) e Zeikus \& Winfrey (1976) a faixa de temperatura ótima para atividade de microorganismos mesófílos na água é de 20 a $45^{\circ} \mathrm{C}$, com valores máximos de produção de $\mathrm{CH}_{4}$ ocorrendo entre 35 a $42^{\circ} \mathrm{C}$. Além da temperatura, a disponibilidade de $\mathrm{N}$ amoniacal (Figura 3) até 250 dias pode ter favorecido o aumento da população microbiana e então da emissão de $\mathrm{CH}_{4}$. 

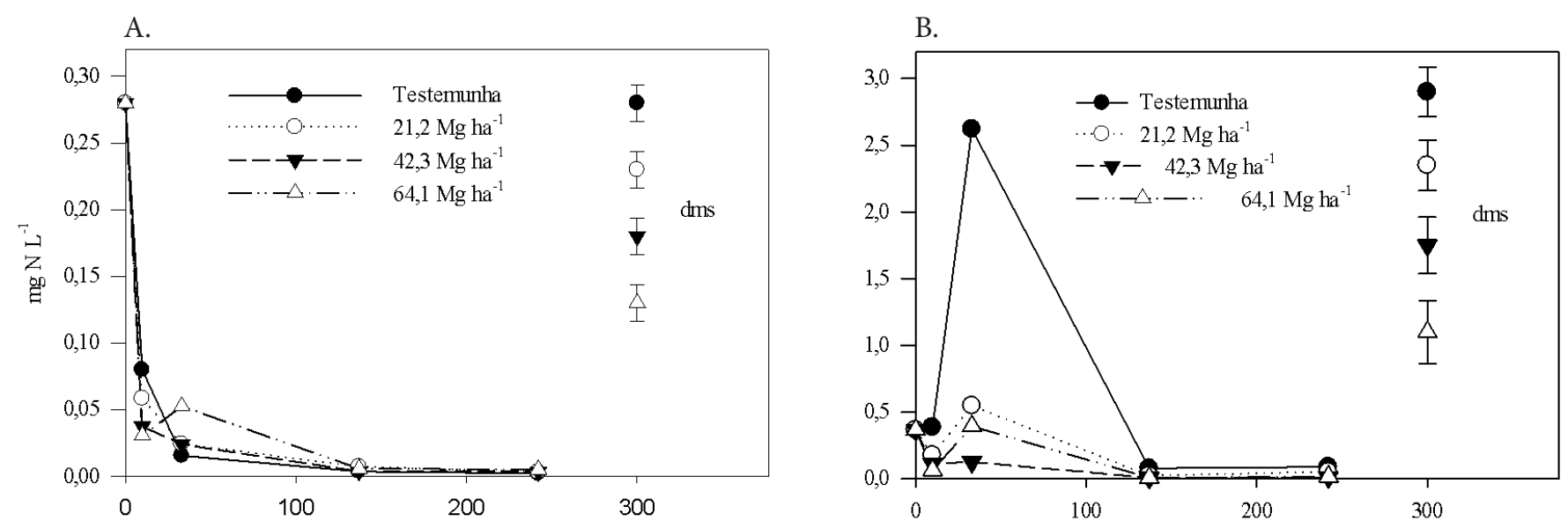

Dias após o alagamento

Figura 2. Comportamento do $\mathrm{N}-\mathrm{NO}_{2}^{-}$(A) e $\mathrm{N}-\mathrm{NO}_{3}^{-}$(B) para cada dose ao longo do tempo e barras ao lado representando a dms entre campanhas

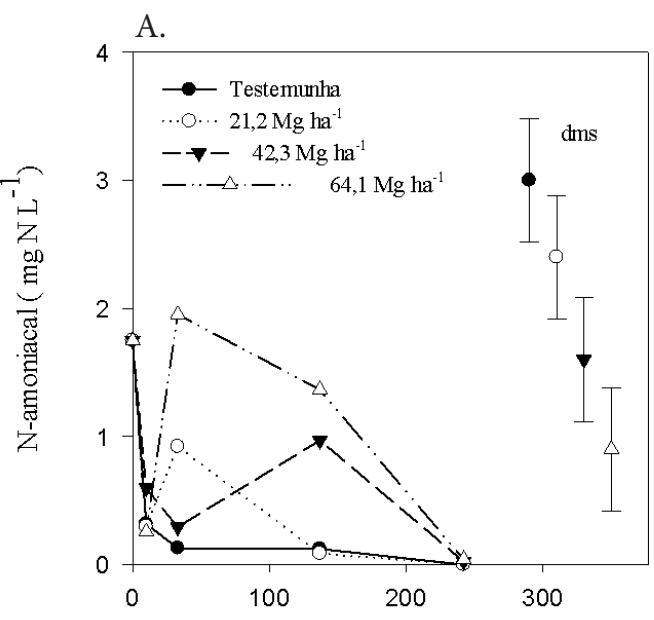

B.

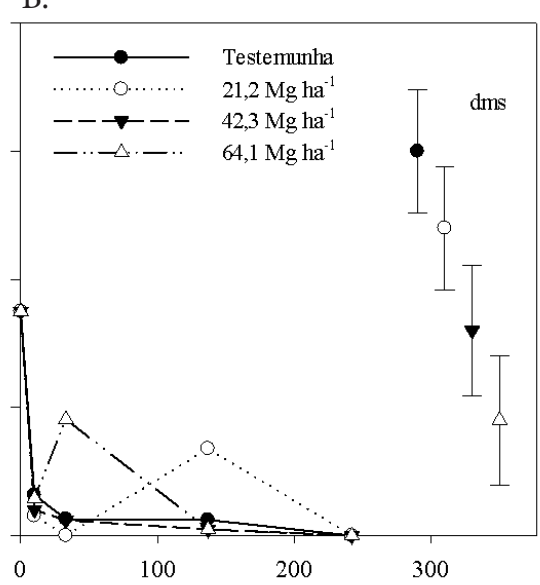

C.

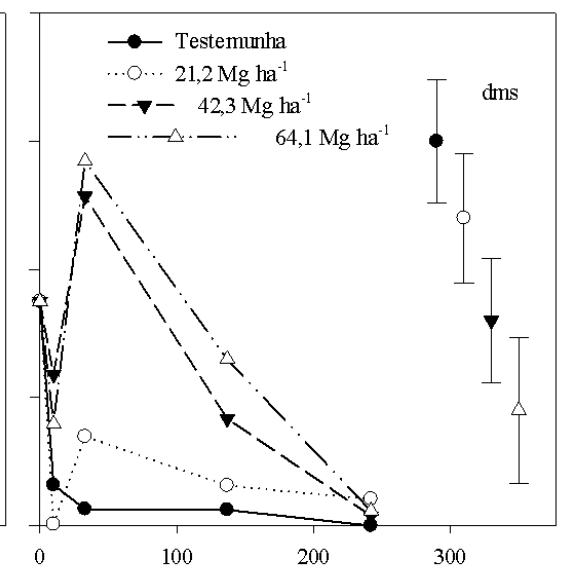

Dias após o alagamento

Figura 3. Comportamento do N-amoniacal ao longo do experimento para o tipo Folhas e Ramos (A), para o tipo Galhos (B), e para o tipo Composição Original (C) com as barras representando dms para cada dose entre campanhas

Em novembro, aos 249 dias após o alagamento, a temperatura do ar foi a maior medida ao longo de todo o experimento $\left(29,3^{\circ} \mathrm{C}\right)$, a qual ocorreu após o período de cinco dias de temperaturas médias diárias acima de $20^{\circ} \mathrm{C}$. Tal combinação deve ter favorecido a produção de $\mathrm{CH}_{4}$, pois no intervalo de 10 a $45^{\circ} \mathrm{C}$ a taxa de crescimento da população microbiana duplica a cada $10^{\circ} \mathrm{C}$ de aumento na temperatura da água (Rittmann \& McCarty, 2001). Em adição, temperaturas maiores que $20{ }^{\circ} \mathrm{C}$ aumentam a solubilização da matéria orgânica (Gujer \& Zehnder, 1983), ao mesmo tempo em que favorecem a produção de $\mathrm{CH}_{4}$ (Bianchini Jr. et al., 2010), confirmando que o processo de metanogênese é mais sensível à variação deste parâmetro se comparado aos demais processos de mineralização (Romeiro \& Bianchini Jr., 2008; Bianchini Jr. et al., 2010).

A emissão acumulada de $\mathrm{CH}_{4}$ ao longo dos 377 dias apresentou médias entre 200, para a testemunha, a 463 g C $\mathrm{m}^{-2}$, para o tipo composição original (Figura 4). Não houve interação entre dose e tipo, havendo efeito somente da dose de resíduo sobre a emissão. A ausência de diferença significativa entre tipos de resíduos pode ter ocorrido pela elevada variabilidade. A alta variação dos resultados é devida, possivelmente, à sensibilidade do processo de metanogênese em resposta a pequenas perturbações no meio (seja em termos de temperatura ou substâncias tóxicas que favoreçam outras rotas de decomposição) (Klüber \& Conrad, 1998; Bianchini et al., 2010).

Embora não haja pontos entre a dose zero e 21,2 Mg $\mathrm{ha}^{-1}$, o modelo exponencial é adequado para explicar o comportamento da emissão acumulada em função da dose. A emissão de $\mathrm{CH}_{4}$ tende a um máximo em função da dose ocorrendo maior diferença somente entre a presença ou não de resíduos visto que todas as doses geraram acumulados médios próximos a $400 \mathrm{~g} \mathrm{C} \mathrm{m}^{-2}\left(404,3 \mathrm{~g} \mathrm{C} \mathrm{m}^{-2}\right.$ - dose menor; 407,5 g C $\mathrm{m}^{-2}$ - dose intermediária e $440,1 \mathrm{~g} \mathrm{C} \mathrm{m}^{-2}$ - dose maior), enquanto que a testemunha gerou uma média próxima da metade deste valor $\left(200 \mathrm{~g} \mathrm{C} \mathrm{m}^{-2}\right)$.

O modelo de regressão tende a um máximo, próximo do valor já alcançado na dose de $60 \mathrm{Mg} \mathrm{ha}^{-1}\left(426 \mathrm{~g} \mathrm{C} \mathrm{m}^{-2}\right)$. Tal máximo estaria relacionado às condições ideais de disponibilização de substrato e liberação de compostos em concentrações que não sejam tóxicas às bactérias metanogênicas. A análise das acumuladas mostra claramente a diferença entre o meio alagado sem resíduos e o meio alagado com os resíduos vegetais. 


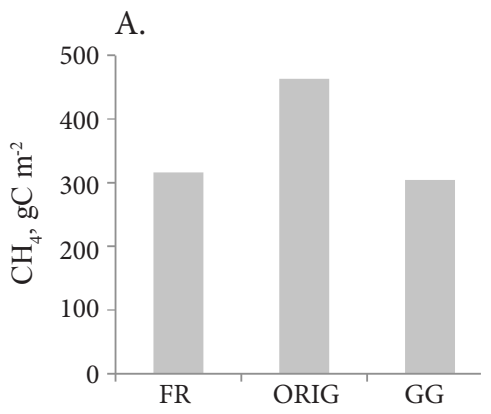

B.

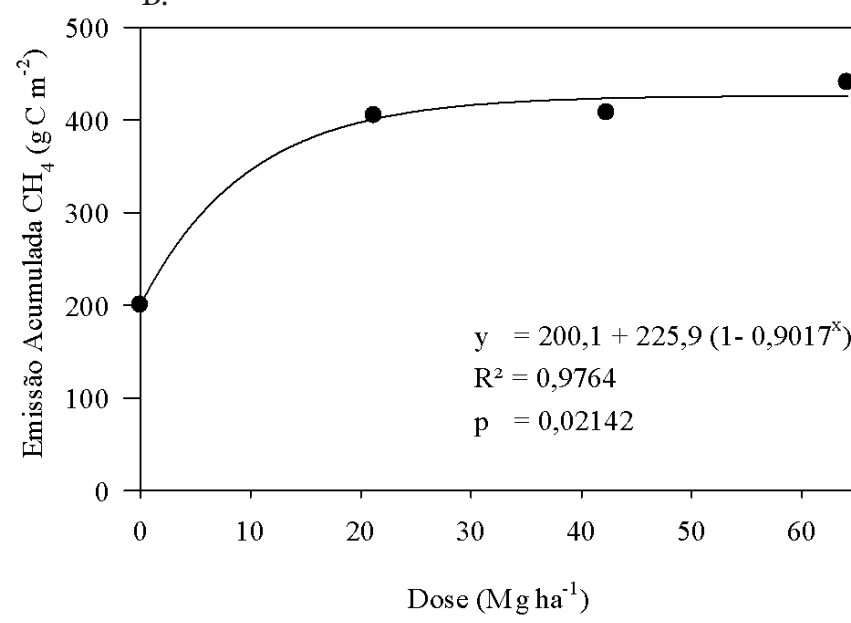

Figura 4. Emissão acumulada de $\mathrm{CH}_{4}$ por tipo de resíduo (FR-folhas e ramos, ORIG-composição original e GGgalhos) e por dose

Em geral, as condições limitantes ou ótimas de emissão estão relacionadas às condições de crescimento da população microbiana. Ainda que haja substrato suficiente para decomposição, esta somente ocorrerá se as condições do meio não forem tóxicas aos micro-organismos e será acentuada quando houver condições ótimas de crescimento e disponibilidade de substrato (Chen et al., 2008). Por sua vez, a comunidade microbiana, associada às condições do meio (íons dissolvidos) está intimamente relacionada às rotas de decomposição do substrato, ou seja, o tipo de bactérias que predominar no meio favorecerá uma ou outra rota de decomposição produzindo, por exemplo, mais $\mathrm{CO}_{2}$ e menos $\mathrm{CH}_{4}$, ou vice-versa (Bianchini Jr. et al., 2010).

\section{Conclusões}

1. A presença de resíduo florestal aumentou a emissão de metano, o que foi visível principalmente pela diferença de emissão entre a testemunha $\left(200 \mathrm{gC} \mathrm{m}^{-2}\right)$ e a dose de $21,2 \mathrm{Mg}$ $\mathrm{ha}^{-1}\left(404 \mathrm{~g} \mathrm{C} \mathrm{m}^{-2}\right)$.

2. O tipo de resíduo florestal, seja este folhas e ramos, galhos ou composição original, não teve efeito sobre a emissão de metano.

3. Houve um tempo de estabilização inicial para a emissão de metano ocorrendo um pico aos 111 dias e outro aos 249 dias após a inundação.

\section{Agradecimentos}

Ao CNPq, pelo benefício concedido através da Lei 8010/90. À Companhia Paranaense de Energia - COPEL, pelo suporte financeiro dentro do programa de P\&D da ANEEL, nos ciclos 2010/2011 e 2011/2012.

\section{Literatura Citada}

Abril, G.; Guérin, F.; Richard, S.; Delmas, R.; Galy-Lacaux, C.; Gosse, P.; Tremblay, A.; Varfalvy, L.; Santos, M. A. \& Matvienko, B. Carbon dioxide and methane emissions and the carbon budget of a 10-year old tropical reservoir (Petit Saut, French Guiana). Global Biogeochemical Cycles, v.19, p.1-16, 2005. http://dx.doi. org/10.1029/2005GB002457

Andalib, M. Nakhla, G., McIntee, E., Zhu, J. Simultaneous denitrification and methanogenesis (SDM): Review of two decades of research. Desalination v, 279, p.1-14, 2011.

Antonio, R. M.; Bianchini Jr., I.. Methodological tests of a heterotrophy index for aquatic ecosystems. Brazilian Journal of Biology,v.63, p.373-380, 2003. http://dx.doi.org/10.1590/S151969842003000300003

APHA - American Public Health Association. Standard Methods for the Examination of Water and Waste Water. 20.ed. Washington, APHA, 1998. 1220p.

Bianchini Jr., I.; Cunha-Santino, M. B. D.; Romeiro, F.; Bitar, A.L. Emissions of methane and carbon dioxide during anaerobic decomposition of aquatic macrophytes from a tropical lagoon (São Paulo, Brazil). Acta Limnologica Brasiliensia, v.22, p.157-164, 2010. http://dx.doi.org/10.1590/S2179$975 \times 2010000200005$

Chen, Y.; Cheng, J. J.; Creamer, K. S. Inhibition of anaerobic digestion process: A review. Bioresource Technology, v.99, p.4044-4064, 2008. http://dx.doi.org/10.1016/j.biortech.2007.01.057

CQFS - Comissão de Química e Fertilidade do Solo - RS/SC. Manual de adubação e de calagem para os Estados do Rio Grande do Sul e Santa Catarina. 10.ed. Porto Alegre: SBCS/ NRS, 2004. 400p.

Das, S.; Adhya, T. K. Dynamics of methanogenesis and methanotrophy in tropical paddy soils as influenced by elevated $\mathrm{CO}_{2}$ and temperature interaction. Soil Biology and Biochemistry, v.47, p.36-45, 2012. http://dx.doi.org/10.1016/j.soilbio.2011.11.020

EMBRAPA - Empresa Brasileira de Pesquisa Agropecuária. Manual de métodos de análises de solo. 2.ed. Rio de Janeiro: MAPA, 1997. 212p.

Fearnside, P. M. Hidrelétricas como "fábricas de metano": O papel dos reservatórios em áreas de floresta tropical na emissão de gases de efeito estufa. Oecologia Brasiliensis, v.12, p.100-115, 2008.

Galy-Lacaux, C.; Delmas, R.; Kouadio, J.; Richard, S. \& Gosse, P. Longterm greenhouse gas emissions from hydroelectric reservoirs in tropical forest regions. Global Biogeochemical Cycles, v.13, p.503517, 1999. http://dx.doi.org/10.1029/1998GB900015

Gimenes, K. Z.; Cunha Santino, M. B. D.; Bianchini Jr., I. Decomposição de matéria orgânica alóctone e autóctone em ecossistemas aquáticos. Oecologia Australis, v.14, p.1036-1073, 2010. http://dx.doi.org/10.4257/oeco.2010.1404.13

Gujer, W.; Zehnder, A. J. B. Conversion processes in anaerobic digestion. Water Science and Technology, v.15, p.127-167, 1983.

Kotzé, J.P.; Thiel, P.G.; Hattingh, W. H. J. Anaerobic digestion II. The characterization and control of anaerobic digestion, Water Research, v.3, p.459-494, 1969. http://dx.doi.org/10.1016/00431354(69)90014-1 
Klüber, H. D.; Conrad, R. Inhibitory effects of nitrate, nitrite, NO and $\mathrm{N}_{2} \mathrm{O}$ on methanogenesis by Methanosarcina barkeri and Methanobacterium bryantii. FEMS Microbiol Ecology, v.25, p.331339, 1998. http://dx.doi.org/10.1016/S0168-6496(97)00102-5

Lee, A. A.; Bukaveckas, P. A. Surface water nutrient concentrations and litter decomposition rates in wetlands impacted by agriculture and mining activities. Aquatic Botany, v.74, p.273-285, 2002. http:// dx.doi.org/10.1016/S0304-3770(02)00128-6

Louis, V. L. St.; Kelly, C. A.; Duchemin, E.; Rudd, J. W. M.; Rosenberg, D. M. Reservoir Surfaces as Sources of Greenhouse Gases to the Atmosphere: A Global Estimate. Bioscience, v.50, p.766-775, 2000. http://dx.doi.org/10.1641/0006-3568(2000)050[0766:RSA SOG]2.0.CO;2

Martins, A. P. L.; Reissmann, C. B. Material vegetal e as rotinas laboratoriais nos procedimentos químico-analíticos. Scientia Agraria, v.8, p.1-17, 2007.

MCT - Ministério da Ciência e Tecnologia. Segunda Comunicação Nacional do Brasil à Convenção-Quadro das Nações Unidas sobre Mudança Global do Clima. Brasília: MCT, 2010. http://www.mct. gov.br/index.php/content/view/326751.html>. 10 Ago. 2011.

Neiff, A. P.; Neiff, J. J.; Casco, S. L. Leaf litter decomposition in three wetland types of the Paraná river floodplain. Wetlands, v.26, p.558566, 2006. http://dx.doi.org/10.1672/0277-5212(2006)26[558:LL DITW]2.0.CO;2

Nelson, D. W.; Sommers, L. E. Total carbon, organic carbon and organic matter. In: Sparks, D. L.; Page A. L.; Helmke, P. A.; Loeppert, R. H.; Soltanpour, P. N.; Tabatabai, M.; Johnston, C. T.; Summer, M. E. (ed.) Methods of soil analysis: Agronomy. Part 2. Madison: Soil Science Society of America, 1996. p.961-1010.
Parkin, T.; Mosier, A.; Smith, J.; Venterea, R.; Johnson, J.; Reicosky, D.; Doyle, G.; Mccarty, G.; Baker, J. GRACEnet Chamberbased trace gas flux measurement protocol. Washington: USDA-ARS, 2003. http://www.usmarc.usda.gov/SP2UserFiles/ person/31831/2003GRACEnetTraceGasProtocol.pdf. 21 Jul. 2011.

Rittmann, B. E.; Mccarty, P. L. Environmental biotechnology. New York: McGraw-Hill, 2001.754p.

Romeiro, F.; Bianchini Jr., I. Kinetic pathways for the anaerobic decomposition of Ludwigia inclinata. Hydrobiologia, v.607, p.103-111, 2008. http://dx.doi.org/10.1007/s10750-008-9370-8

Rudd, J. W. M.; Harris, R.; Kelly, C. A.; Hecky, R. E. Are hydroelectric reservoirs significant sources of greenhouse gases? Ambio, v.22, p.246-248, 1993.

Santos, M. A.; Rosa, L. P.; Matvienko, B.; Santos, E. O.; Rocha, C. D.; Sikar, E.; Silva, M. B.; Júnior, M. A. Emissões de gases de efeito estufa por reservatórios de hidrelétricas. Oecologia Brasiliensis, v.12, p.116-129, 2008.

Sbrissia, R. C.; Fernandes, C. V. S.; Braga, M. C. B.; Santos A. F. dos. Estimativa de emissões de gases de efeito estufa em reservatórios a partir da dinâmica da matéria orgânica na coluna da água: Estudo de caso PCH Salto Natal, Campo Mourão - Paraná. Revista Brasileira de Recursos Hídricos, v.16, p.59-69, 2011.

Soest, P. J. van; Wine, R. H. The determination of lignin and cellulose in acid-detergent fiber with permanganate. Journal of the Association of Official Analytical Chemists, v.52, p.780-785, 1968.

Zeikus, J. G.; Winfrey, M. R. Temperature limitation of methanogenesis in aquatic sediments. Applied and Environmental Microbiology, v.31, p.99-107, 1976. 